\title{
In search of the optimal setting for statin trials in prostate cancer: the power of population-based studies
}

\author{
Emma H. Allott $\mathbb{B}^{1,2} \cdot$ Emma L. Craig ${ }^{1} \cdot$ Konrad H. Stopsack $\mathbb{B}^{3}$
}

Received: 18 February 2021 / Revised: 8 March 2021 / Accepted: 11 March 2021 / Published online: 29 March 2021

(c) The Author(s), under exclusive licence to Springer Nature Limited 2021

Mounting evidence from epidemiological studies suggests a potential role for statins in lowering the risk of aggressive and lethal prostate cancer, without a clear association between statin use and the risk of low-risk prostate cancer. Moreover, several registry-based studies have demonstrated lower prostate cancer-specific mortality among men already diagnosed with prostate cancer who use statins, suggesting a potential benefit even after cancer diagnosis [1]. Randomized-controlled trials of statins in a post-diagnosis setting could provide much-needed evidence for considering implementation of statins into clinical care of men with prostate cancer. Yet, more work needs to be done in identifying patient subgroups who are most likely to benefit. The findings from Peltomaa et al. [2] suggest that a potential setting for such a trial may be among men undergoing androgen deprivation therapy (ADT).

Within the Finnish Randomized Study of Screening for Prostate Cancer, part of the larger European Randomized Study of Screening for Prostate Cancer, the authors studied 4428 men with prostate cancer diagnosed between 1996 and 2015 who received ADT, most as part of primary therapy. With 834 prostate cancer deaths occurring during a median follow-up of 6.3 years from ADT initiation, statin use after ADT initiation was associated with a lower risk of PSA recurrence (HR, 0.73 ; 95\% CI 0.65-0.82) and less prostate cancer-specific mortality (HR, $0.82 ; 95 \%$ CI $0.69-0.96$ ), with a stronger association among men using higher statin

Konrad H. Stopsack

stopsack@mskcc.org

1 Patrick G. Johnston Centre for Cancer Research, Queen's University Belfast, Belfast, Northern Ireland, UK

2 Department of Histopathology and Morbid Anatomy, Trinity Translational Medicine Institute, Trinity College Dublin, Dublin, Ireland

3 Department of Medicine, Memorial Sloan Kettering Cancer Center, New York, NY, USA amounts. By contrast, statin use before starting ADT or at the time of starting ADT was not associated with prostate cancer outcomes. The authors propose that their results contribute evidence for a synergistic effect between statins and ADT.

Support for this hypothesis comes from earlier observations by this same group of researchers on an overlapping study population [3]. The original study showed a somewhat stronger inverse association between post-diagnosis statin use and prostate cancer-specific mortality among men who received ADT, compared to those undergoing surgery or radiotherapy. Moreover, a number of other observational studies [4-6] reported lower prostate cancer-specific mortality in ADT-treated men who were also using statins. Like some of these previous studies, the current study cannot definitively conclude that there is a stronger association between statin use and lower prostate cancer mortality due to concomitant ADT, given that all men received ADT. However, there is a strong biological rationale behind this hypothesis: the prostate accumulates cholesterol ester stores which, by serving as the precursor for de novo steroidogenesis, can furnish sufficient androgens to support the growing tumor even with castrate circulating androgen levels achieved by ADT [7]. Thus, statins may contribute to lowering intratumoral androgens, thereby improving response to ADT. Experimental studies in the $\mathrm{LNCaP}$ xenograft mouse model of prostate cancer support this hypothesis. Using surgical castration, one study reported reduced tumor growth and lower intratumoural androgens following simvastatin treatment [8], while another showed slower tumor growth, enhanced apoptosis, and lower proliferation indices following atorvastatin treatment [9].

It is the power of population-based observational studies that allows researchers like Peltomaa and colleagues to examine whether these observations made in the lab might translate into clinical benefit for humans, before launching randomized trials. Registry and linkage systems allowed the capture of virtually the entire population of interest; the study was large enough to assess less common but 
important outcomes like prostate cancer death; detailed exposure data on statin prescriptions could be incorporated; and data on important confounders could be leveraged.

Another powerful aspect of the current study is that it uses the toolkit of epidemiology to probe whether the observed associations could be causal. By defining the exposure, statin use, as a time-dependent variable, the authors took the first step to reducing one of the major sources of bias, immortal time bias-which can occur if analysts overlook that getting a statin prescription implies that a study participant must be alive at that time point. Another form of selection bias, reverse causation, could arise if men stopped taking non-cancer medications shortly before a death from cancer. In lagged analyses where the investigators defined statin exposure as usage in the preceding year, the association between statin use and prostate cancer mortality was somewhat attenuated (HR, 0.89; $95 \%$ CI 0.76-1.04). These estimates do not fully allow ruling out reverse causation as one potential contributor to the observed results.

As always with non-randomized exposures, confounding is a consideration, in pharmacoepidemiology studies particularly confounding by indication. Confounding becomes more challenging to address when confounders are mainly measured at baseline but the exposure changes over time, as is the case for statin use after ADT initiation. It is possible that time-varying confounding explains some of the observed lower prostate cancer death rates when defining statin exposure as use after ADT initiation, but not when defining it before ADT initiation (HR, 1.13; 95\% CI $1.02-1.25)$ or in the first year on ADT (HR, 1.02; 95\% CI, $0.85-1.24)$. An alternative, more favorable explanation for the observed inverse association of statin use measured post-ADT but not pre-ADT is that statins would need to be continued throughout treatment because their effects would simply not last for several years after cessation, or indeed that statin use synergizes with ADT treatment by suppressing intratumoral androgens.

The power of observational studies ends, at least for therapy questions, when deciding whether treatments should become standard of care. Randomized-controlled trials are needed. Such trials are laborious, costly, and typically do not give results for several years. Since statins are generic, such trials may need to be run by academic investigators [10]. Yet one further argument for statins in the setting of ADT should not be forgotten: the high cardiovascular disease risk among men with advanced prostate cancer. Given that statins have proven cardiovascular benefit, the use of these safe drugs by men with prostate cancer could have a dual benefit. In the study by Peltomaa et al., nearly half of the men used statins at some point during follow-up. The question of whether the other men would benefit from statins deserves to be answered in randomizedcontrolled trials.

Acknowledgements This work was funded in part by a National Cancer Institute Cancer Center Support Grant (P30CA008748), the Irish Cancer Society (John Fitzpatrick Fellowship to EHA), the Department of Defense (Early-Investigator Research Award W81XWH-18-1-0330 to KHS), and the Prostate Cancer Foundation (Young Investigator Award to KHS).

Publisher's note Springer Nature remains neutral with regard to jurisdictional claims in published maps and institutional affiliations.

\section{References}

1. Alfaqih MA, Allott EH, Hamilton RJ, Freeman MR, Freedland SJ. The current evidence on statin use and prostate cancer prevention: are we there yet? Nat Rev Urol. 2017;14:107-19.

2. Peltomaa AI, Raittinen P, Talala K, Taari K, Tammela TL, Auvinen A, et al. Prostate cancer prognosis after initiation of androgen deprivation therapy among statin users. A populationbased cohort study. Prostate Cancer Prostatic Dis. 2021.

3. Murtola TJ, Peltomaa AI, Talala K, Maattanen L, Taari K, Tammela TLJ, et al. Statin use and prostate cancer survival in the finnish randomized study of screening for prostate cancer. Eur Urol Focus. 2017;3:212-20.

4. Hamilton RJ, Ding K, Crook JM, O'Callaghan CJ, Higano CS, Dearnaley DP, et al. The association between statin use and outcomes in patients initiating androgen deprivation therapy. Eur Urol. 2021;79:446-52.

5. Harshman LC, Wang X, Nakabayashi M, Xie W, Valenca L, Werner L, et al. Statin use at the time of initiation of androgen deprivation therapy and time to progression in patients with hormone-sensitive prostate cancer. JAMA Oncol. 2015;1: 495-504.

6. Larsen SB, Dehlendorff C, Skriver C, Dalton SO, Jespersen CG, Borre M, et al. Postdiagnosis statin use and mortality in danish patients with prostate cancer. J Clin Oncol. 2017;35:3290-7.

7. Montgomery RB, Mostaghel EA, Vessella R, Hess DL, Kalhorn $\mathrm{TF}$, Higano CS, et al. Maintenance of intratumoral androgens in metastatic prostate cancer: a mechanism for castration-resistant tumor growth. Cancer Res. 2008;68:4447-54.

8. Gordon JA, Midha A, Szeitz A, Ghaffari M, Adomat HH, Guo Y, et al. Oral simvastatin administration delays castration-resistant progression and reduces intratumoral steroidogenesis of $\mathrm{LNCaP}$ prostate cancer xenografts. Prostate Cancer Prostatic Dis. 2016;19: 21-7.

9. Zheng X, Cui XX, Gao Z, Zhao Y, Lin Y, Shih WJ, et al. Atorvastatin and celecoxib in combination inhibits the progression of androgen-dependent $\mathrm{LNCaP}$ xenograft prostate tumors to androgen independence. Cancer Prev Res (Phila). 2010;3:114-24.

10. Murtola TJ, Syvala H, Tolonen T, Helminen M, Riikonen J, Koskimaki J, et al. Atorvastatin versus placebo for prostate cancer before radical prostatectomy-a randomized, double-blind, placebo-controlled clinical trial. Eur Urol. 2018;74:697-701. 